\title{
CHARACTERISTIC CLASSES AND DISTORTION OF DIFFEOMORPHISMS
}

\author{
OLIVER ATTIE, JONATHAN BLOCK, AND SHMUEL WEINBERGER
}

Let $M$ and $N$ be manifolds of bounded geometry; that is the absolute value of their curvatures, as well as their covariant derivatives, are bounded from above and their injectivity radii are bounded from below. A map $f: M \rightarrow N$ has bounded distortion if there is an upper bound on the norm of the derivative $D f$. A bounded distortion diffeomorphism (b.d.d.) is assumed to have an inverse which also has bounded distortion. Similarly, homotopies are assumed to have bounded distortion. Note that this implies that homotopic maps are a finite distance apart.

We prove the following theorem that describes when characteristic classes of a compact manifold obstruct the existence of bounded distortion diffeomorphisms of universal covers.

Theorem. There are compact manifolds $A$ and $B$ with fundamental group $\Gamma$ and a map $f: A \rightarrow B$ with $f^{*}(p(B)) \neq p(A)$ for some rational Pontrjagin class $p$, whose universal cover is b.d. homotopic to a b.d. diffeomorphism if and only if $\Gamma$ is nonamenable.

In the first author's forthcoming thesis much more precise analyses will be given for $\Gamma$ abelian.

Proof. First we prove necessity; that is, for $\Gamma$ amenable no such maps exist. Let $M$ be a manifold of bounded geometry. We define $H_{\beta}^{i}(M)$ as in [3] to be the DeRham cohomology groups based on differential forms $\omega$ such that $\omega$ and $d \omega$ are uniformly bounded. The Pontrjagin forms of a manifold of bounded geometry live in this group. Bounded distortion maps induce maps on $H_{\beta}^{i}(M)$. The groups are therefore homotopy invariants in the category of bounded geometry manifolds and such maps. In particular, the Pontrjagin forms give well-defined elements of $H_{\beta}^{i}(M)$ for the manifold up to b.d.d. The necessity follows from the following:

Proposition. If the fundamental group of $A$ is amenable, then the pullback induces an injection $H^{i}(A) \rightarrow H_{\beta}^{i}(\tilde{A})$ where $\tilde{A}$ denotes the universal cover of $A$.

This is an extension of "Nonexample $0.2 \mathrm{~A}$ " of [2].

Received by the editors August 29, 1991.

1991 Mathematics Subject Classification. Primary 57R20, 57R50; Secondary 58B30.

The second author was partially supported by an NSF postdoctoral fellowship. The third author was partially supported by an NSF grant and a PYI award. 
Proof. If the fundamental group of $A$ is amenable, $\tilde{A}$ has a regular exhaustion $U_{i}$ consisting of unions of fundamental domains where

(1) $U_{i} \subset U_{i+1}$

(2) $\cup_{i} U_{i}=\tilde{A}$ and

(3) one has ${ }^{1}$ vol $\partial U_{i} /$ vol $U_{i} \rightarrow 0$.

By taking a weak-* limit of the functionals on bounded $n$-forms

$$
\omega \mapsto \frac{1}{\operatorname{vol} U_{i}} \int_{U_{i}} \omega
$$

one obtains a homomorphism $H_{\beta}^{n}(\tilde{A}) \rightarrow \mathbb{R}$, see [3]. This homomorphism takes the pullback of the volume form to vol $A$ and is thus nontrivial. Since pullback preserves wedge product, Poincaré duality yields the result: for any nonzero cohomology class $[\varphi]$ let $\varphi$ be a harmonic representative. Then $\varphi \wedge * \varphi$ is a positive multiple of the volume form. On pulling up to the universal cover, their pairing is still nontrivial since it is detected by the functional, proving the proposition.

To prove the opposite direction, we will use the "Ponzi scheme" introduced by the last two authors in [1] as well as a little bit of surgery theory (see e.g. [5]).

First for the construction of the manifolds. Let $M$ be any compact $m$ manifold with the given fundamental group. Let $V$ be any simply connected $v$-manifold such that $H_{i}(V ; \mathbb{Q})$ is nonzero for some $i=-m \bmod 4, i \leq v-1$. The manifold $A$ is simply $V \times M$. Under these assumptions, surgery theory [5] produces a manifold $W$ with a homotopy equivalence $W \rightarrow V \times D^{m}$ that restricts to a diffeomorphism on the boundary and is detected by relative Pontrjagin classes. (The aggregate of such $W$, up to rel boundary diffeomorphism, form a group under "stacking" which is abelian for $m \geq 2$, and is computed by the surgery exact sequence, which is, in this case, a sequence of abelian groups and homomorphisms.) $B$ is obtained by "grafting" $W$ into $A: \quad B=\left(A-\operatorname{int}\left(V \times D^{m}\right)\right) \cup W$. There is an obvious map $B \rightarrow A$, and by construction, it does not pull back Pontrjagin classes.

Now we verify that this works. Let $[W]$ denote the map $W \rightarrow V \times D^{m}$. Consider the adjacency graph of the tiling of the universal cover $\tilde{A}$ by fundamental domains. The Ponzi scheme of [1] consists of integers $a_{e}$ for each oriented edge $e$ of the graph satisfying three conditions:

(1) The $a_{e}$ are uniformly bounded in size,

(2) if $e$ and $-e$ are the same edge oriented oppositely, then $a_{e}=-a_{-e}$, and

(3) if we sum $a_{e}$ over all the edges going into a fixed vertex, the sum is always -1 .

We can modify $\tilde{B}$ by grafting $a_{e}[W]$ along each oriented edge $e$. By condition (1) this is still naturally a manifold of bounded geometry. By condition (2) it is, in fact, b.d.d. to $\tilde{B}$. Finally, using condition (3) and the construction of

\footnotetext{
${ }^{1}$ This is the only really critical property
} 
$B$ as a grafted $A$, one can reorganize the graftings around each vertex (i.e. in a neighborhood of each fudamental domain) to see that this manifold is b.d.d. also to $\tilde{A}$. This completes the proof.

Remark. As long as $\Gamma$ is infinite one can obtain a Ponzi scheme that violates condition (1). ${ }^{2}$ This means that if we do the analogous construction in the amenable case, the universal cover of the map does remain a bounded distance from a diffeomorphism, but this diffeomorphism will necessarily have distortion growing as one approaches infinity.

\section{ACKNOWLEDGMENTS}

The second two authors would like to thank the Courant Institute for its hospitality while this paper was written.

\section{REFERENCES}

1. J. Block and S. Weinberger, Aperiodic tilings, positive scalar curvature, and amenability of spaces, J. Amer. Math. Soc. 5 (1992), 907-918.

2. M. Gromov, Kahler-hyperbolicity and $L^{2}$-Hodge theory, J. Differential Geom. 33 (1991), 263-292.

3. J. Roe, An index theorem on open manifolds. I, II, J. Differential Geom. 27 (1988), 87-136.

4. J. Stallings, On infinite processes leading to differentiability in the complement of a point, Differential and Combinatorial Topology (S. S. Cairns, ed.), Princeton Univ. Press, Princeton, NJ, 1965, pp. 245-254.

5. C.T.C. Wall, Surgery on compact manifolds, Academic Press, San Diego, CA, 1971.

Courant Institute of the Mathematical Sciences, New York University, New York, NEW YORK 10012

E-mail address: attie@acf9.nyu.edu

Mathematics Department, University of Pennsylvania, Philadelphia, Pennsylvania 19104

E-mail address: blockj@math.upenn.edu

Mathematics Department, University of Chicago, Chicago, Illinois 60607

E-mail address: shmuel@zaphod.uchicago.edu

\footnotetext{
${ }^{2}$ Such Ponzi schemes without boundedness conditions are often referred to as Eilenberg swindles; see [4] for other geometric applications of this idea.
} 